\title{
Porcine diazepam-binding inhibitor and bovine diazepam-binding inhibitor affect morphine antinociception via different receptors
}

\section{Dear Editor,}

Opioid addiction is one of the top challenges for society, particularly in China. To fight it, the key is to reveal its underlying mechanisms. Among the strategies to overcome the mental damage caused by opioids, investigating native anti-opioid peptides derived from mammalian (including human) brains is an important option because of safety concerns. In 1983, diazepam-binding inhibitor (DBI), a 10-kDa peptide, was first derived from rat brains (Guidotti et al., 1983). After repeated treatment with morphine, the DBI level is enhanced in rodent brains (Katsura et al., 1998; Shibasaki et al., 2006).

In the present work, we report an anti-opioid peptide-99A (AOP-99A) sample purified from porcine brain, the sequence of which is the same as that of the known DBI from pig intestine that reduces glucose-induced insulin secretion (Chen et al., 1988). We named it pDBI (Fig. 1A and 1B). Purified pDBI showed a single band on SDS-PAGE (Fig. 1C) and had a molecular weight of $9791 \mathrm{Da}$ as determined by mass spectrometry (Fig. S1). Morphine antinociception was suppressed by purified pDBI ( $2.0 \mathrm{nmol} / \mathrm{L}, 4 \mu \mathrm{L}$ i.c.v., Fig. $1 \mathrm{D})$.

There is known to be $92 \%$ homology between the aminoacid residues of pDBI/AOP-99A and bovine DBI/endozepine (or bDBI, Figs. 1A, S2). The different amino residues of the two DBI-subfamilies are at positions $6,14,22,46,49,60$, and 75 from the N-terminal. Western blotting showed a positive immuno-response of $\mathrm{pDBI}$ to $\mathrm{McAb}-\mathrm{B} 2$ against $\mathrm{pDBI}$, but not bovine or rat $\mathrm{DBI}$. This showed that $\mathrm{pDBI}$ differs from bDBI (Figs. $1 C$ and S3). By degradation of $\mathrm{pDBI}$ with trypsin and separation by micro-HPLC, pDBI was separated into 12 peaks (Fig. 1E). Among these, the samples from peaks 10 and 11 substantially suppressed morphine antinociception (Fig. 1F).

In order to determine the sequences of the samples from peaks 10 and 11 , two peptides pDBI-16 (99A-16) and pDBI19 (99A-19) were synthesized based on residues 17-32 and 53-71 (Fig. 1A). Functional experiments showed that morphine antinociception was suppressed by i.c.v. injection of $\mathrm{pDBI}-16(2.5,5.0$, and $10 \mathrm{nmol} / \mathrm{L}$, Fig. S4) and pDBI-19 (2.5, 5.0, and $10 \mathrm{nmol} / \mathrm{L}$, Fig. 1G). Thus, we found that the effect of $\mathrm{pDBI}-16$ and $\mathrm{pDBI}-19$ on morphine antinociception was same as that of full-length $\mathrm{pDBI}$.

In order to reveal the bioactivity of bDBI-16 and bDBI-19, the fragments consistent with the 17-32 and 53-71 residues in $\mathrm{bDBI}$ were synthesized (Fig. 1A). Like full-length $\mathrm{pDBI}$, pDBI-16, pDBI-19, and bDBI-16 (10, 20, and $40 \mathrm{nmol} / \mathrm{L})$ also had a suppressive effect (Figs. 2A, C, and S3). However, the morphine antinociceptive effect was potentiated by bDBI-19 (Fig. 2B and 2D). This effect was similar to the potentiating effect of bDBI (i.c.v. in the $2-4 \mathrm{nmol} / \mathrm{L}$ dose range) on morphine antinociception (Chen et al., 1991).

$\mathrm{DBI}$ is a $10-\mathrm{kDa}$ polypeptide that was first isolated by monitoring its ability to displace diazepam from the benzodiazepine (BDZ) recognition site located on the extracellular domain of the $G_{A B A}$ receptor and on the mitochondrial membrane (Guidotti et al., 1983; Costa and Guidotti, 1991). BDZ receptors are classified into two types: a central type linked to the $G A B A_{A}$ receptor-chloride channel complex, and a peripheral type not linked to the $\mathrm{GABA}_{\mathrm{A}}$ receptor (Costa and Guidotti, 1991). The peripheral type is expressed at high levels on the outer mitochondrial membrane, particularly in astrocytes (Anholt et al., 1986; Costa and Guidotti, 1991). When mice were pretreated with flumazenil, an antagonist of the central type of BDZ receptor, the suppressed morphine antinociception was still induced by i.c.v. injection of saline, pDBI (2.0 nmol/L), pDBI-16 (10 nmol/L), pDBI-19 (10 nmol/L), and $\mathrm{bDBI}-16(40 \mathrm{nmol} / \mathrm{L})$. To our surprise, the potentiated morphine antinociception induced by bDBI-19 (13.6 nmol/L) was reversed in flumazenil-pretreated mice (Fig. 2E). However, in mice pretreated with PK-11195, an antagonist of the peripheral type of BDZ receptor, the suppressed morphine antinociception induced by i.c.v. injection of $\mathrm{pDBI}(2.0 \mathrm{nmol} / \mathrm{L})$, pDBI-16 (10.0 nmol/L), pDBI-19 (10.0 nmol/L), and bDBI-16 $(40 \mathrm{nmol} / \mathrm{L})$ was lost (Fig. 2F). Our experiments, for the first time, revealed that the anti-morphine effect of $p D B l s$ and $\mathrm{bDBI}-16$ is mediated by the peripheral type of $\mathrm{BDZ}$ receptor, while the morphine-enhancing effect of bDBI-19 is mediated by the central type of $\mathrm{BDZ}$ receptor (Fig. 2E and 2F). There is a long lasting open question how i.v. bDBI can produce its enhancing-morphine effect same as by i.c.v. bDBI (Chen et al., $1991 \mathrm{~b})$, because bDBI $(10 \mathrm{kDa})$ is too large to permeate the 


\section{A}

bDBI: SQAEFDKAAE EVKHLKTKPA DEEMLFIYSH YKQATVGDIN TERPGMLDFK GKAKWDAWNE LKGTSKEDAM KAYIDKVEEL KKKYGI bDBI-16: TKPADEEMLFIYSHYK

bDBI-19:

AKWDAWNELKGTSKEDAMK

PDBI: SOAEFEKAAE EVKNLKTKPA DDEMLFIYSH YKOATVGDIN TERPGILDLK GKAKWDAWWG LKGTSKEDAM KAYINKVEEL KKKYGI pDBI-16: TKPADDEMLFIYSHYK

pDBI-19: AKWDAWNGLKGTSKEDAMK

B

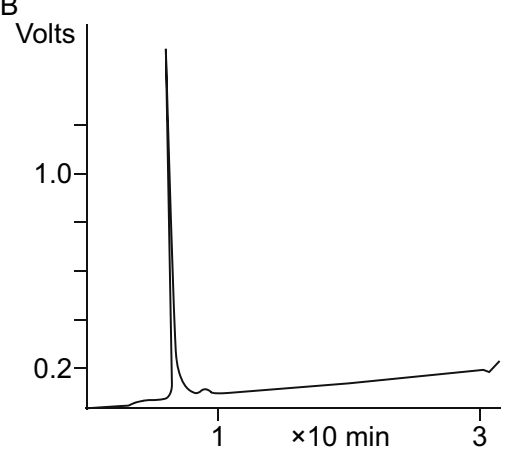

C

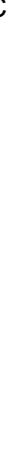

AKWDAWNGLKGTSKEDAMK

The
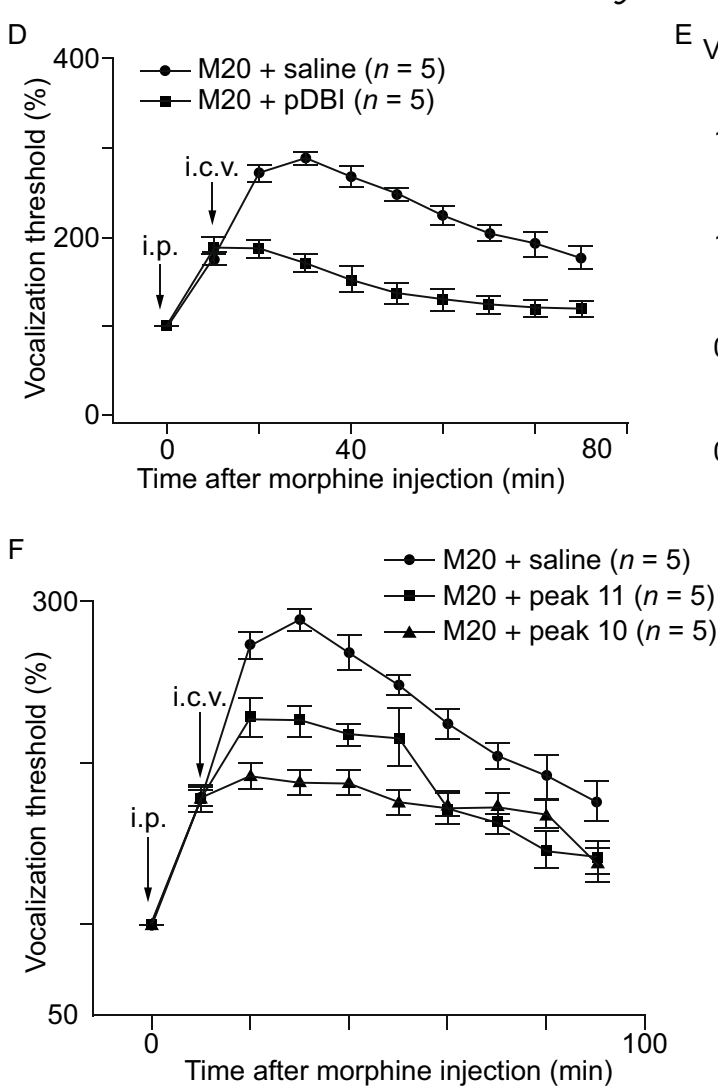
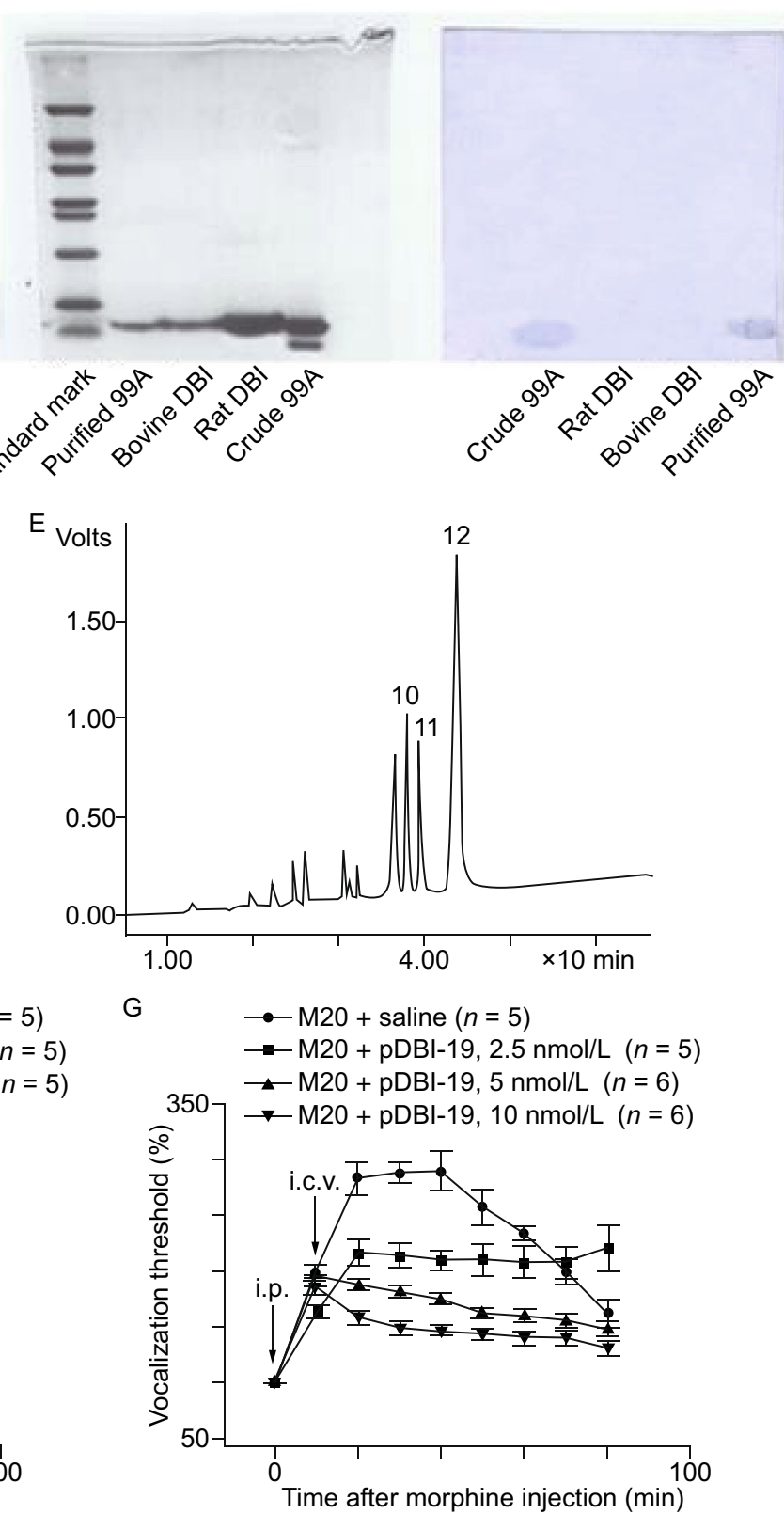

Figure 1. Structures of porcine $\mathrm{DBI}$, bovine $\mathrm{DBI}$, and their active fragments. (A) Schematic of the amino-acid sequences of $\mathrm{pDBI}$ and bDBI peptides and the four active fragments. (B) pDBI purified by HPLC on a C18-reverse-phase column. (C) pDBI showed a single band on SDS-PAGE (12\%) and semi-dry western blotting. (D) Effect of pDBI (2 nmol/L, i.c.v.) on morphine antinociception (20 $\mathrm{mg} / \mathrm{kg}$, i.p.) at different time. Comparation of control/saline $(n=5)$ and $\mathrm{pDBI}$ groups $(n=5), P<0.01$, one-way ANOVA, followed by student's t-test. (E) Hydrolysate of pDBI with trypsin separated by reversed-phase HPLC. (F) Bioactivity of samples from peaks 11 and 10. Comparation of saline, peak 10, and peak 11 groups $(n=5), P<0.01$. (G) Effect of pDBI-19 on morphine antinociception 10 min after morphine administration (20 mg/kg, i.p.). Comparation of saline, pDBI-19 (2.5 nmol/L), pDBI-19 (5.0 nmol/L), and pDBI-19 $(10 \mathrm{nmol} / \mathrm{L})$ groups $(n=6), P<0.01$. 
A

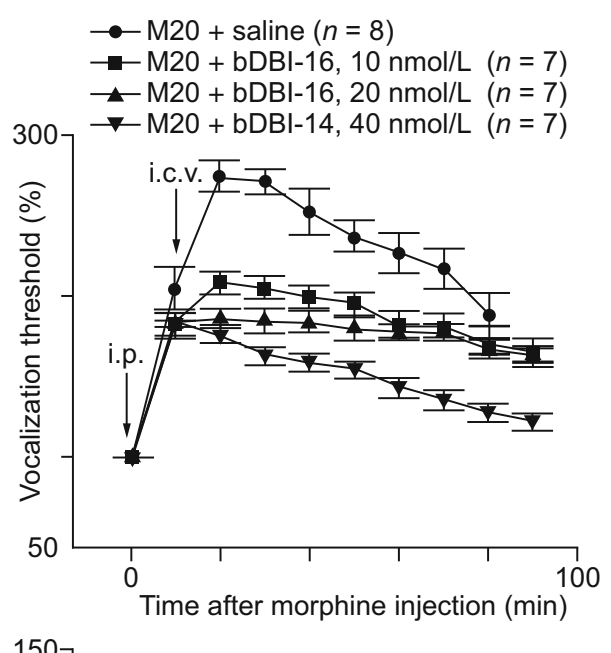

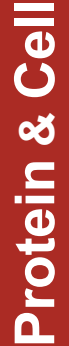
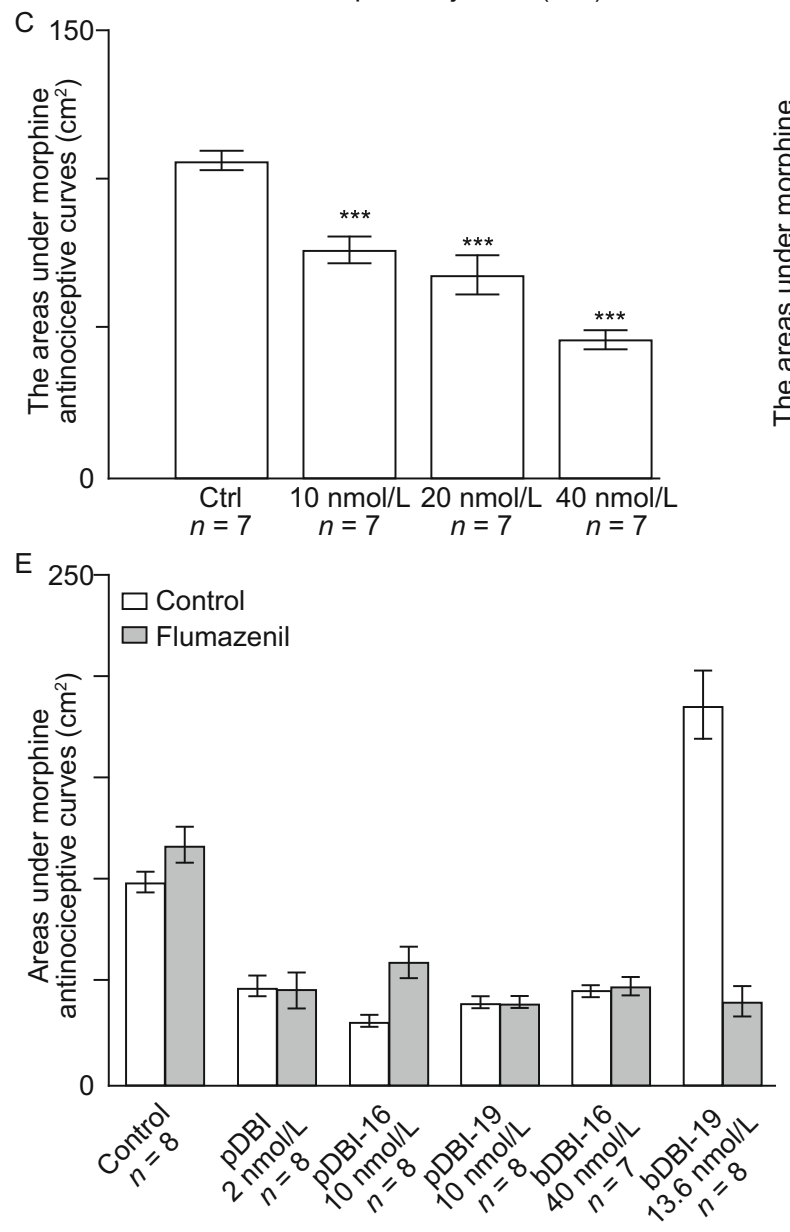
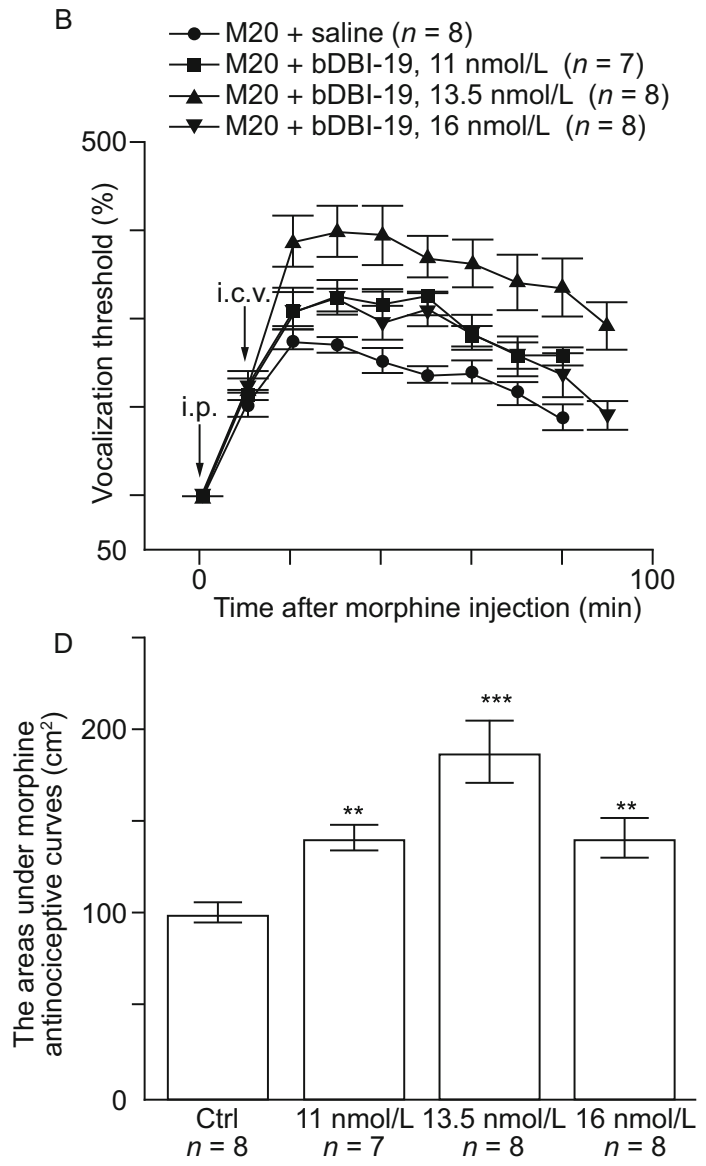

$\mathrm{F}$

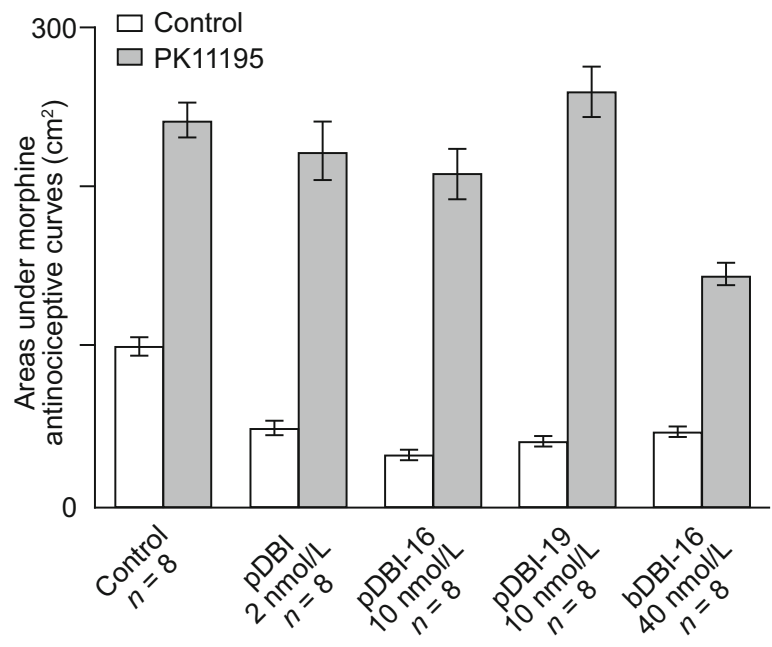

Figure 2. Two functional subtypes of $D B I$ peptides and morphine-antinociception: enhancer versus inhibitor. (A) Morphine antinociception was significantly suppressed by bDBI-16 (i.c.v.). Comparation of saline, bDBI-16 (10 nmol/L), bDBI-16 (20 nmol/L), and bDBI-16 (40 nmol/L) groups $(n=7) 10 \mathrm{~min}$ after morphine injection (20 mg/kg, i.p.). (B) Potentiating effect of bDBI-19 on morphine antinociception. (C) Dose-response curve for suppressed morphine antinociception induced by bDBI-16 $\left({ }^{\star \star} P<0.05\right.$, ${ }^{* * *} P<0.001$ compared to control). (D) Dose-response curve for potentiated morphine antinociception induced by bDBI-19 ${ }^{* *} P<0.05$, ${ }^{* * *} P<0.001$ compared to control). (E) In mice treated with flumazenil, the morphine antinociception induced by pDBI, pDBI-16, pDBI19 , and bDBI-16 was still suppressed, but the potentiated morphine antinociception induced by bDBI-19 was reversed $(n=8$, $P<0.001$, student's t-test). (F) In mice treated with PK11195, the suppressed morphine antinociception induced by pDBI, pDBI-16, pDBI-19, and bDBI-16 was fully removed. 
BBB, which permeates substance $<3 \mathrm{kDa}$ only. This puzzle is now solved, because intravenous bDBI is degraded in the serum into smaller peptides, including the blood-brain barrierpermeable bDBI-19 $(2 \mathrm{kDa})$, which produces the bDBI-like enhancing-morphine effect (Fig. 2B and 2D).

We propose that the opposite effects of full-length pDBI and bDBI on morphine antinociception (Chen et al., 1991) are due to the opposing hydrophobicity of residues at position 60 and the different types of BDZ receptors. Interestingly, bDBI potentiates morphine effect of antinociception, but its 2 active fragments bDBI-16 and bDBI-19 show opposite effects (Figs. 2 and S4). The underlying mechanisms are unclear. Future work is needed to address the remaining questions (1) whether the peripheral type of BDZ receptor is sensitive to bDBI-19, and (2) why bDBI-16 has an effect opposite to bDBI and bDBI-19.

It is known that the amino-acid DBI-E60 is conserved in rat, mouse, cow, and human (Costa and Guidotti, 1991) but not in porcine $\mathrm{DBI}$ derived from pig intestine. The function of $\mathrm{pDBI}$ and its fragments (pDBI-33-50 and pDBI 17-50) are associated with various neuropsychiatric disorders involving neuronal excitability (Costa and Guidotti, 1991). In the present work, we discovered that pDBI has two active fragments (pDBI-16 and pDBI-19) that suppress morphine antinociception. Thus, pDBls might play important roles during morphine tolerance and dependence (data not shown). It is known that DBI concentration increases in mammals following continuous treatment with morphine (Katsura et al., 1998; Shibasaki et al., 2006). Therefore, we suggest that $\mathrm{pDBI}$ and $\mathrm{bDBI}$ and the active fragments may co-exist in the central nervous system and play multiple physiological roles during the development of morphine tolerance and dependence. Although it remains unclear how pDBls and bDBls are produced after morphine intake in humans, our study provides a new mechanism of morphine tolerance and dependence underlying addiction in animals which may help future efforts treating addicted patients.

\section{ACKNOWLEDGEMENTS}

We thank Dr. lain $C$ Bruce for helpful comments on the manuscript. ZZ and ZL were partially supported by grants from the National Basic Research Program (973 Program) (No. 2012CB518006), and the National Natural Science Foundation of China (Grant Nos. 31228010, 31171026, 31100597, 31327901, 31221002, 31330024, 31400708, 31670843, 31521062, and SQ2011SF11B01041).

Yu-Zhen Chen, Xiao-Cun Li, Zhen-Quan Guo, Li Zhou, Zhuan Zhou, Song-vve no conflict of interest. This article does not contain any studies with human subjects performed by the any of the authors. All institutional and national guidelines for the care and use of laboratory animals were followed.

Cai-Hong Wu-Deceased

Electronic supplementary material The online version of this article (doi:10.1007/s13238-016-0355-5) contains supplementary material, which is available to authorized users.
Yu-Zhen Chen ${ }^{1,4 凶}$, Xiao-Cun $\mathrm{Li}^{1,2}$, Zhen-Quan Guo ${ }^{2}$, Li Zhou ${ }^{1,3}$, Zhuan Zhou ${ }^{1,3 凶}$, Song-Ping Liang ${ }^{5}$, Cai-Hong $\mathrm{Wu}^{1,2}$

${ }^{1}$ State Key Laboratory of Membrane Biology, Peking University, Beijing 100871, China

${ }^{2}$ College of Life Sciences, Peking University, Beijing 100871, China

${ }^{3}$ PKU-IDG/McGovern Institute for Brain Research and Beijing Key Laboratory of Cardiometabolic Molecular Medicine, Institute of Molecular Medicine and Peking-Tsinghua Center for Life Sciences, Peking University, Beijing 100871, China

${ }^{4}$ Institute of Clinical Medical Science, China-Japan Friendship Hospital, Beijing 100029, China

${ }^{5}$ College of Life Sciences, Hunan Normal University, Changsha 410006, Hunan, China

$\triangle$ Correspondence: chenyuzhen2016pku@163.com (Y.-Z. Chen), zzhou@pku.edu.cn (Z. Zhou)

\section{OPEN ACCESS}

This article is distributed under the terms of the Creative Commons Attribution 4.0 International License (http://creativecommons.org/ licenses/by/4.0/), which permits unrestricted use, distribution, and reproduction in any medium, provided you give appropriate credit to the original author(s) and the source, provide a link to the Creative Commons license, and indicate if changes were made.

\section{REFERENCES}

Anholt RR, Pedersen PL, De Souza EB, Snyder SH (1986) The peripheral-type benzodiazepine receptor. Localization to the mitochondrial outer membrane. J Biol Chem 261:576-583

Chen ZW, Agerberth B, Gell K, Andersson M, Mutt V, Ostenson CG, Efendic S, Barros-Soderling J, Persson B, Jornvall H (1988) Isolation and characterization of porcine diazepam-binding inhibitor, a polypeptide not only of cerebral occurrence but also common in intestinal tissues and with effects on regulation of insulin release. Eur J Biochem 174:239-245

Chen YZ, Wang JY, Zhou S, Shoyab M (1991) Bovine endozepine potentiates morphine analgesia in mice. Life Sci 48:PL79-PL83

Costa E, Guidotti A (1991) Diazepam binding inhibitor (DBI): a peptide with multiple biological actions. Life Sci 49:325-344

Guidotti A, Forchetti CM, Corda MG, Konkel D, Bennett CD, Costa E (1983) Isolation, characterization, and purification to homogeneity of an endogenous polypeptide with agonistic action on benzodiazepine receptors. Proc Natl Acad Sci USA 80:3531-3535

Katsura M, Hara A, Higo A, Tarumi C, Hibino Y, Ohkuma S (1998) Continuous treatment with morphine increases diazepam binding inhibitor mRNA in mouse brain. J Neurochem 71:2638-2641

Shibasaki M, Katsura M, Tsujimura A, Ohkuma S (2006) Upregulated L-type high voltage-gated calcium channels cause increase in diazepam binding inhibitor induced by sustained morphine exposure in mouse cerebrocortical neurons. Life Sci 80:166-172 